\title{
A Coastal Cline in Sodium Accumulation in Arabidopsis thaliana Is Driven by Natural Variation of the Sodium Transporter AtHKT1;1
}

\author{
Ivan Baxter ${ }^{19}$, Jessica N. Brazelton ${ }^{29}$, Danni $\mathrm{Yu}^{3}$, Yu S. Huang ${ }^{4}$, Brett Lahner ${ }^{2}$, Elena Yakubova ${ }^{2}$, Yan $\mathrm{Li}^{5}$, \\ Joy Bergelson ${ }^{5}$, Justin O. Borevitz ${ }^{5}$, Magnus Nordborg ${ }^{6}$, Olga Vitek ${ }^{3}$, David E. Salt ${ }^{2 *}$
}

1 United States Department of Agriculture-Agricultural Research Service, Plant Genetics Research Unit, Donald Danforth Plant Sciences Center, St. Louis, Missouri, United States of America, 2 Department of Horticulture and Landscape Architecture, Purdue University, West Lafayette, Indiana, United States of America, 3 Department of Statistics, Purdue University, West Lafayette, Indiana, United States of America, $\mathbf{4}$ Molecular and Computational Biology, University of Southern California, Los Angeles, California, United States of America, 5 Department of Ecology and Evolution, University of Chicago, Chicago, Illinois, United States of America, 6 Gregor Mendel Institute of Molecular Plant Biology, Austrian Academy of Sciences, Vienna, Austria

\begin{abstract}
The genetic model plant Arabidopsis thaliana, like many plant species, experiences a range of edaphic conditions across its natural habitat. Such heterogeneity may drive local adaptation, though the molecular genetic basis remains elusive. Here, we describe a study in which we used genome-wide association mapping, genetic complementation, and gene expression studies to identify cis-regulatory expression level polymorphisms at the AtHKT1;1 locus, encoding a known sodium ( $\mathrm{Na}^{+}$) transporter, as being a major factor controlling natural variation in leaf $\mathrm{Na}^{+}$accumulation capacity across the global $A$. thaliana population. A weak allele of $A t H K T 1 ; 1$ that drives elevated leaf $\mathrm{Na}^{+}$in this population has been previously linked to elevated salinity tolerance. Inspection of the geographical distribution of this allele revealed its significant enrichment in populations associated with the coast and saline soils in Europe. The fixation of this weak AtHKT1;1 allele in these populations is genetic evidence supporting local adaptation to these potentially saline impacted environments.
\end{abstract}

Citation: Baxter I, Brazelton JN, Yu D, Huang YS, Lahner B, et al. (2010) A Coastal Cline in Sodium Accumulation in Arabidopsis thaliana Is Driven by Natura Variation of the Sodium Transporter AtHKT1;1. PLoS Genet 6(11): e1001193. doi:10.1371/journal.pgen.1001193

Editor: Gregory P. Copenhaver, The University of North Carolina at Chapel Hill, United States of America

Received August 2, 2010; Accepted October 1, 2010; Published November 11, 2010

This is an open-access article distributed under the terms of the Creative Commons Attribution License, which permits unrestricted use, distribution, and reproduction in any medium, provided the original author and source are credited.

Funding: This work was supported by the US National Institutes of Health (http://www.nih.gov/) grants R01 GM078536 and 3R01GM078536-03S1 and by Purdue University Center for Gene-Environment Interactions to DES. The funders had no role in study design, data collection and analysis, decision to publish, or preparation of the manuscript.

Competing Interests: The authors have declared that no competing interests exist.

* E-mail: dsalt@purdue.edu

9 These authors contributed equally to this work.

\section{Introduction}

Uncovering the genetic polymorphisms that underlie adaptation to environmental gradients is a critical goal in evolutionary biology, and will lead to a better understanding of both the types of genetic changes and the gene functions involved. Such understanding will not only provide insight into how organisms may respond to future global climate change, but will also provide tools for the development of agricultural systems and ecological services that are more resilient to such changes.

Patterns of phenotypic diversity across environmental gradients can be indicative of adaptive responses to selection, and evaluation of these patterns has the potential to lead to the identification of the genetic polymorphisms underlying these adaptive responses. Numerous studies in animals and plants have identified phenotypic clines in various life history traits, but only a few have determined the genetic changes driving such traits. In Arabidopsis thaliana, plasticity in seasonally regulated flowering appears to be modulated by a network of gene interactions responsive to both vernalization and photoperiod signals [1]. Adaptive clines in resistance to oxidative stress and chilling [2], and wing size [3] in Drosophila melanogaster are modulated by the Insulin-like Receptor (InR) and Drosophila cold acclimation (Dca) genes, respectively. While adaptation to high altitude in Peromyscus maniculatus (Deer mice) is associated with enhanced pulmonary $\mathrm{O}_{2}$ loading driven by alterations in $\alpha$-globin and $\beta$-globin genes [4]. These genetic changes are all associated with adaptation to variation in environmental factors that vary with latitude or altitude. Such systematic variation has greatly facilitated the discovery of these loci and their adaptive significance. Clines in various life history traits have also been identified in plants growing on serpentine [5], saline [6,7], and mine impacted soils [8]. Progress has been made in outlining the genetic architecture of these adaptive traits [5,8-10], though a molecular genetic understanding is still needed.

A. thaliana is broadly distributed in its native Europe and central Asia, where it experiences a wide range of altitudinal, climatic, and edaphic conditions, leading to a range of selective pressures [11]. Whether the wide variety of natural phenotypic and genetic variation observed in A. thaliana [12] contributes to its local adaptation is an important unresolved question that is currently attracting a significant amount of attention [13].

Because of its relevance to crop production, salinity tolerance in plants has been studied intensively [14], and natural plant populations adapted to such conditions have provided an excellent 


\section{Author Summary}

The unusual geographical distribution of certain animal and plant species has provided puzzling questions to the scientific community regarding the interrelationship of evolutionary and geographic histories for generations. With DNA sequencing, such puzzles have now extended to the geographical distribution of genetic variation within a species. Here, we explain one such puzzle in the European population of Arabidopsis thaliana, where we find that a version of a gene encoding for a sodium-transporter with reduced function is almost uniquely found in populations of this plant growing close to the coast or on known saline soils. This version of the gene has previously been linked with elevated salinity tolerance, and its unusual distribution in populations of plants growing in coastal regions and on saline soils suggests that it is playing a role in adapting these plants to the elevated salinity of their local environment.

system for studying the evolutionary mechanisms of adaptation and speciation in coastal $[6,10]$ and salt marsh [7,9,15-18] environments. The primary effects of excess $\mathrm{Na}^{+}$on plants are water deficit resulting from a water potential gradient between the soil solution and plant cells, and cytotoxicity due of intracellular $\mathrm{Na}^{+}$accumulation [14]. To overcome these effects plants must both accumulate solutes for osmotic regulation, and detoxify intracellular $\mathrm{Na}^{+}$either by limiting its accumulation, or by compartmentalizing $\mathrm{Na}^{+}$into the vacuole. In addition, $\mathrm{Na}^{+}$ compartmentalization facilitates vacuolar osmotic adjustment that is necessary to compensate for the osmotic effects of salinity by maintaining turgor pressure for cell expansion and growth. Plants therefore need to strike a balance between the accumulation of $\mathrm{Na}^{+}$to maintain turgor, and the need to avoid $\mathrm{Na}^{+}$chemical toxicity, and this balance will depend in part on soil salinity levels. Given the critical role $\mathrm{Na}^{+}$accumulation plays in salinity tolerance, we used this life history trait to probe the global $A$. thaliana population for signals of adaptive selection for growth in saline impacted environments.

\section{Results/Discussion}

We grew 349 accessions of $A$. thaliana in a controlled common garden in non-saline soil, and analyzed leaf $\mathrm{Na}^{+}$accumulation. We observed a wide range of leaf $\mathrm{Na}^{+}$accumulation across the accessions $\left(330-4,848 \mathrm{mg} \mathrm{kg}^{-1}\right.$ dry weight). If this natural variation in leaf $\mathrm{Na}^{+}$accumulation capacity is related to adaptation to growth in saline soils we would expect to find evidence of an adaptive cline, or a gradient of leaf $\mathrm{Na}^{+}$ accumulation that correlates with the geographical distribution of variation in soil salinity. Salinity impacted soils are expected to occur in coastal regions due to air born deposition of sea spray which can occur many tens of $\mathrm{km}$ inland [19-22], but can also occur in areas distant from the coast through high $\mathrm{Na}^{+}$in the soil or ground water. Elevated soil salinity can also be caused by inappropriate irrigation practices such as irrigation with saline water or poor drainage.

To test for the existence of an adaptive cline in leaf $\mathrm{Na}^{+}$ accumulation capacity and soil salinity we related leaf $\mathrm{Na}^{+}$ accumulation capacity to the distance of the collection site for each accession to the coast, or to the nearest known saline soil, whichever is the shortest. We focused on European accessions since a good soil salinity map exists for this region [23], which left 300 accessions. Regressing the distance to the coast, or nearest known saline soil, on leaf $\mathrm{Na}^{+}$for all 300 accessions revealed a significant relationship ( $\mathrm{p}$-value $<2 \mathrm{e}-12$ ), establishing that accessions with elevated leaf $\mathrm{Na}^{+}$are more likely to grow in potentially saline impacted soils (Figure 1A and 1B).

To investigate the genetic architecture underlying this cline in leaf $\mathrm{Na}^{+}$accumulation capacity we performed a genome-wide association (GWA) study (previously described for a smaller data set [24]) to identify regions of the genome at which genetic variation is associated with leaf $\mathrm{Na}^{+}$accumulation capacity. The 337 A. thatiana accessions used in our GWA study, which are a subset of the 349 accessions phenotyped for leaf $\mathrm{Na}^{+}$, were genotyped using the Affymetrix SNP-tilling array Atsnptilela which can interrogate 248,584 SNPs. To assess evidence of association between SNPs and leaf $\mathrm{Na}^{+}$accumulation we used a mixed-model approach [25] to correct for population structure, as previously described [24]. In the current analysis we identified a single strong peak of SNPs associated with leaf $\mathrm{Na}^{+}$, with the peak centered on AtHKT1;1 (Figure 2), a gene known to encode a $\mathrm{Na}^{+}$transporter [26]. Accessions with a thymine (T) at the SNP most significantly associated with leaf $\mathrm{Na}^{+}$at position $6392276 \mathrm{bp}$ on chromosome 4 (Chr4:6392276) have significantly higher leaf $\mathrm{Na}^{+}$ than accessions with a cytosine $(\mathbf{C})$ at this same position (2,325 vs. $955 \mathrm{mg} \mathrm{Na}^{+} \mathrm{kg}^{-1}$ dry weight, $\mathrm{p}$-value $\left.<2 \mathrm{e}-16\right)$. This SNP explains $32 \%$ (without accounting for population structure) of the total variation in leaf $\mathrm{Na}^{+}$accumulation observed.

Previously, in independent test crosses between the high leaf $\mathrm{Na}^{+}$accessions Ts-1 and Tsu-1 (both containing a $\mathrm{T}$ at Chr4:6392276) and the low leaf $\mathrm{Na}^{+}$accession Col-0 (containing a $\mathrm{C}$ at Chr4:6392276) QTLs for leaf $\mathrm{Na}^{+}$centered on AtHKT1;1 were identified in both F2 populations [27]. Such genetic evidence provides independent support that the peak of SNPs associated with leaf $\mathrm{Na}^{+}$observed in our GWA analysis, centered at AtHKT1;1 (Figure 2), represents a true positive association and not a false positive driven by the high degree of population structure known to exist in $A$. thaliana [24]. Reduced expression of AtHKT 1; 1 in Ts-1 and Tsu-1 was concluded to drive the elevated leaf $\mathrm{Na}^{+}$observed in these two accessions [27]. Here, we expand on this observation by establishing the strength of the AtHKT 1;1 alleles in four further high $\mathrm{Na}^{+}$accumulating accessions (Bur-1, Duk, PHW-20 and UKNW06-386) that all contain a $\mathrm{T}$ at Chr4:6392276, along with a low leaf $\mathrm{Na}^{+}$accession (Nd-1) with a $\mathrm{C}$ at Chr4:6392276. By examining the leaf $\mathrm{Na}^{+}$accumulation in $\mathrm{F} 1$ plants from crosses of each of these accessions to Col- $0^{h k t 1-1}$ and Col- $0^{\mathrm{HKT} 1}$, we were able to establish a significant correlation between leaf $\mathrm{Na}^{+}$accumulation and the strength of the AtHKT1;1 alleles (Figure 3A). These crosses confirmed that all accessions tested with elevated leaf $\mathrm{Na}^{+}$, and that contain a $\mathrm{T}$ at Chr4:6392276, have hypofunctional alleles of AtHKT1;1 relative to the Col-0 allele. Furthermore, analysis of the expression of AtHKT 1;1 in the same set of accessions revealed that allelic variation in AtHKT1;1 strength is modulated at the level of gene expression (Figure 3B), consistent with what was previously observed for Ts-1 and Tsu-1 [27]. Though the SNP most significantly associated with leaf $\mathrm{Na}^{+}$(Chr4:6392276) is unlikely to be causal for these AtHKT1;1 expression level polymorphisms, this SNP can be used as a linked genetic marker to determine the type of AtHKT1;1 allele present, with a $\mathrm{T}$ at this SNP being associated with weak AtHKT1;1 alleles.

Using the SNP at Chr4:6392276 as a genetic marker for the type of AtHKT1;1 allele (strong or weak) allowed us to test the hypothesis that the leaf $\mathrm{Na}^{+}$soil salinity cline we observe in European populations of $A$. thaliana (Figure $1 \mathrm{~A}$ and $1 \mathrm{~B}$ ) is associated with weak alleles of AtHKT1;1. By comparing the means of distances to the coast, or known saline soil, for the 


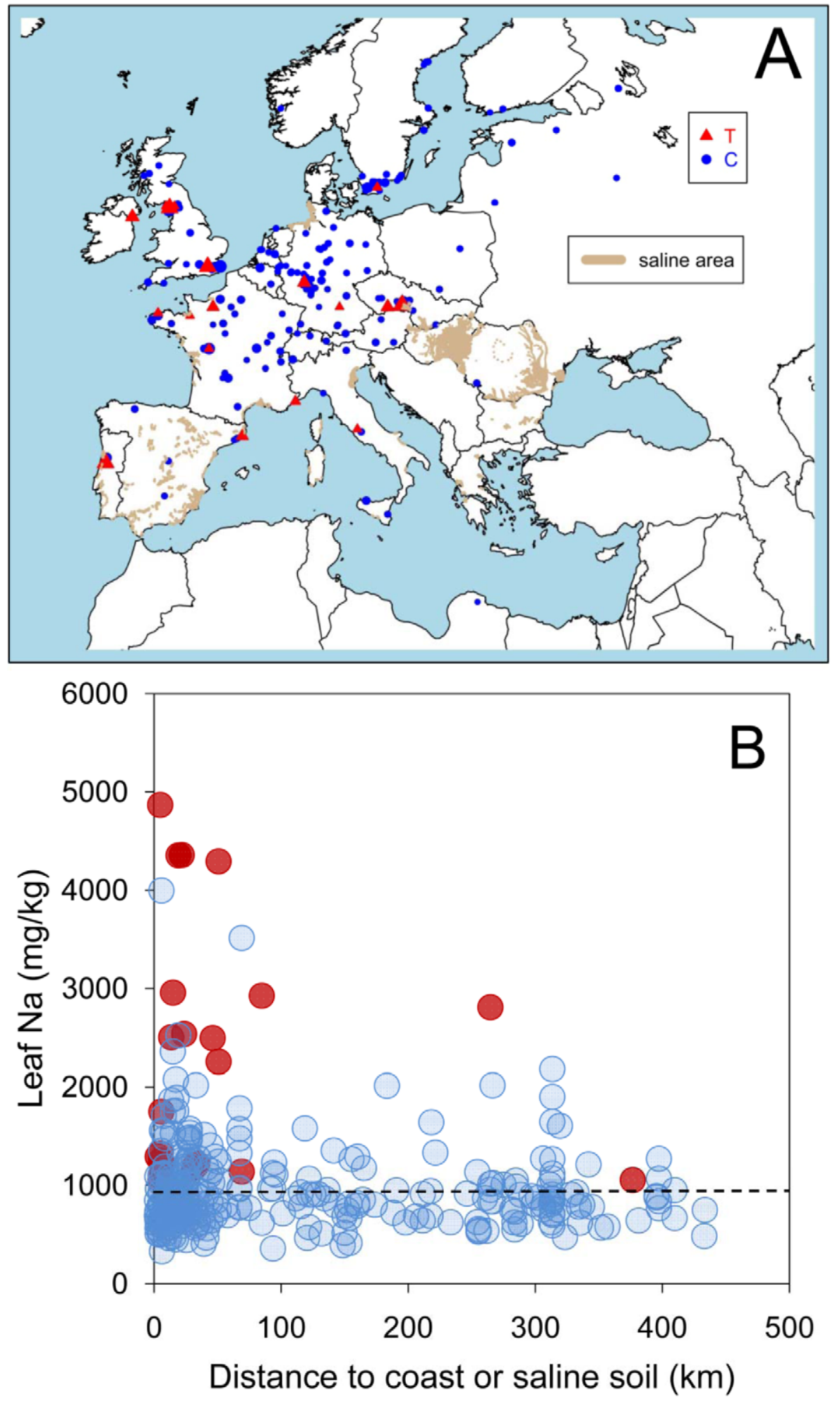

Figure 1. A coastal/saline soil cline in leaf $\mathrm{Na}^{+}$accumulation in $A$. thaliana driven by natural variation at the AtHKT1; 1 locus. A. Map showing the geographical position for the collection site of 300 accessions of $A$. thaliana. The genotype at Chr4:6392276 of each accession is represented by the type of symbol (red triangle Chr4:6392276 = T, blue circle Chr4:6392276=C). Leaf Na ${ }^{+}$accumulation of each accession, measured in a common garden experiment, is represented by the size of the symbols. Known areas of saline soils were obtained from Tóth et al., [23] and are represented here in green. B. Leaf $\mathrm{Na}^{+}$accumulation in 300 accessions of $A$. thaliana, measured in a common garden experiment, and its relationship with the distance to the collection site of each accession to the coast or known saline soils (whichever is the smallest). Red symbols genotype at Chr4:6392276 = T, blue symbols genotype at Chr4:6392276 = C. Dashed black line represents the average leaf $\mathrm{Na}^{+}$for the Col-0 accession. doi:10.1371/journal.pgen.1001193.g001 


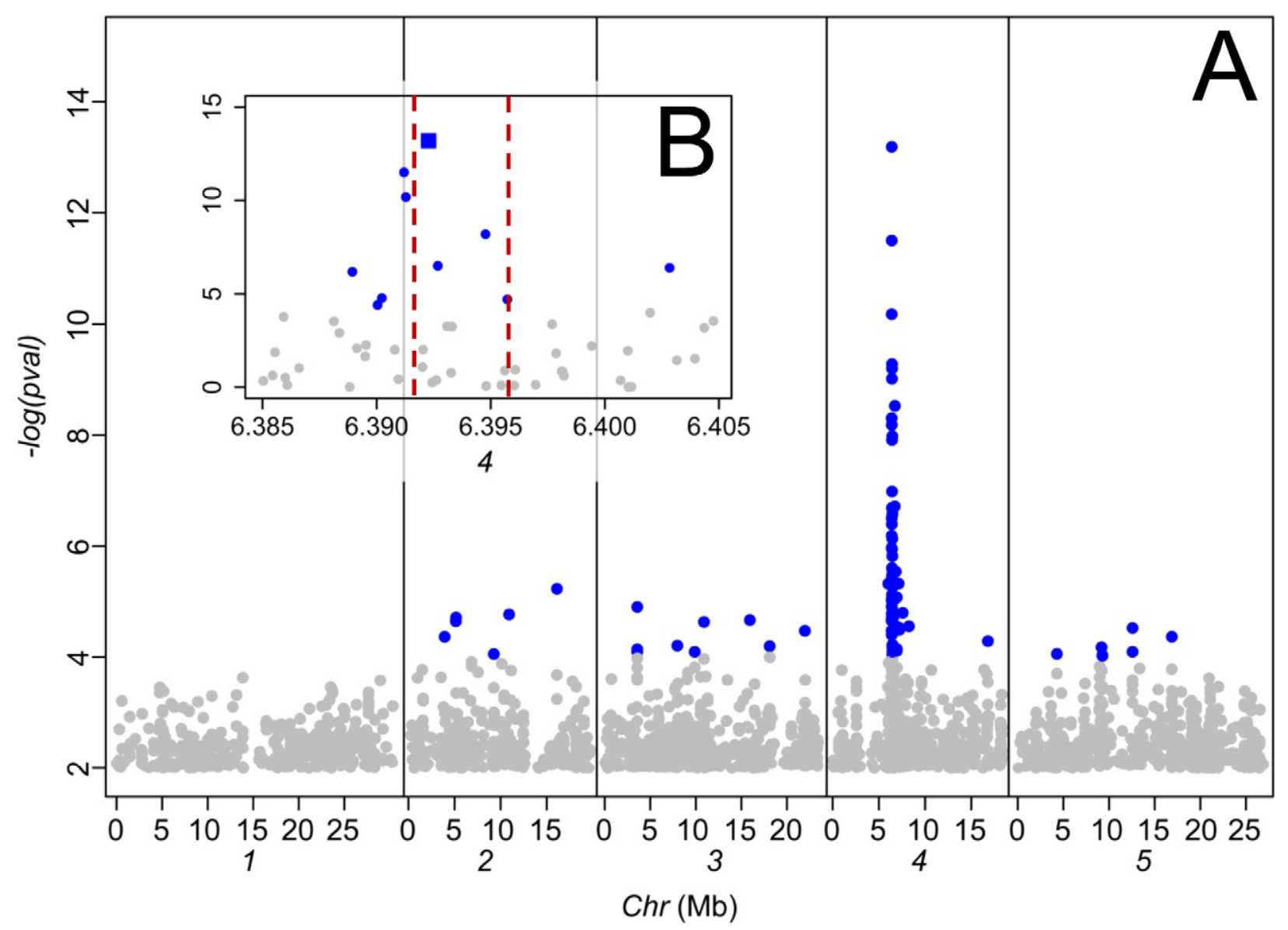

Figure 2. Genome-wide association analysis of leaf $\mathrm{Na}^{+}$accumulation in 349 A. thaliana accessions grown in a common garden. A. Genome-wide $p$-values from a mixed model analysis implemented in EMMA [24]. Associations with a $p$-value $>0.001$ are shaded in grey. B. Magnification of the genomic region surrounding AtHKT1;1, the position and extent of which is indicated by vertical lines (locus At4g10310 Chr4:6391984-6395877). The SNP most significantly associated with leaf $\mathrm{Na}^{+}$accumulation (Chr4:6392276) is represented by a square symbol, all other SNPs represented by circles. Mb, megabase.

doi:10.1371/journal.pgen.1001193.g002

collection site of all 300 accessions with and without a $\mathrm{T}$ at Chr4:6392276, we determined that a significant association (parametric test $\mathrm{p}$-value $=0.0001$; non-parametric Wilcoxon rank-sum test $\mathrm{p}$-value $=0.0062$ ) exists between $A$. thaliana growing on potentially saline impacted soils and the presence of a weak allele of AtHKT1;1 (Figure 1A and 1B). Such a strong correlation between the presence of allelic variation at AtHKT1;1 known to drive elevated leaf $\mathrm{Na}^{+}$, and the observed cline in leaf $\mathrm{Na}^{+}$and saline soils, is evidence for the involvement of AtHKT1;1 in determining this geographical distribution. Furthermore, using 13 SNPs within a $20 \mathrm{~kb}$ region centered on HKT1;1 to define the HKT 1;1 haplotype, we identify 7 haplotypes (6 if you combine haplotypes with only 1 SNP different) in accessions with high leaf $\mathrm{Na}^{+}(>2,500 \mathrm{ppm})$, suggesting that weak alleles of HKT1;1 have arisen independently multiple times.

However, to be credible it is also important to provide evidence that selection for growth on saline soils could be acting on the phenotype driven by allelic variation at AtHKT1; 1 in this case elevated leaf $\mathrm{Na}^{+}$. Such evidence is provided by the previous observation that the weak allele of AtHKT1;1 in the coastal Tsu-1 A. thatiana accession not only causes elevated leaf $\mathrm{Na}^{+}$but is also genetically linked to the elevated salinity tolerance of this accession [27].

In A. thaliana AtHKT1;1 functions to unload $\mathrm{Na}^{+}$from xylem vessels in the root, controlling translocation and accumulation of
$\mathrm{Na}^{+}$in the shoots $[26,28]$. Therefore, modulation of its function would allow the balancing of $\mathrm{Na}^{+}$accumulation in the shoot with soil salinity. We note here that the hkt1-1 null mutation in the Col0 background causes plants to exhibit dramatic leaf $\mathrm{Na}^{+}$ hyperaccumulation and increased $\mathrm{NaCl}$ sensitivity [29,30]. We interpret this to mean that expression of AtHKT1;1 in the hkt1-1 null mutant is reduced to such an extent that leaf $\mathrm{Na}^{+}$ accumulation saturates the capacity for cellular detoxification of $\mathrm{Na}^{+}$by vacuolar compartmentalization. We propose that the naturally occurring weak alleles of AtHKT1;1, that we show are associated with populations growing in potentially saline impacted environments, allow sufficient $\mathrm{Na}^{+}$to accumulate in leaves for osmotic adjustment, conferring elevated $\mathrm{Na}^{+}$tolerance. However, these weak, but not complete loss-of-function AtHKT1;1 alleles, do not saturate the mechanism whereby the accessions avoid $\mathrm{Na}^{+}$ cytotoxicity. The basis of this $\mathrm{Na}^{+}$detoxification mechanism remains to be determined, though an active leaf vacuolar $\mathrm{Na}^{+}$ compartmentalization mechanism driven by AtNHX1 is one likely candidate.

In conclusion, here we provide evidence supporting the involvement of specific cis-regulatory polymorphisms at AtHKT1;1 in the potentially adaptive cline in leaf $\mathrm{Na}^{+}$accumulation capacity we observe in $A$. thaliana populations to saline impacted environments. We have identified a strong association between 

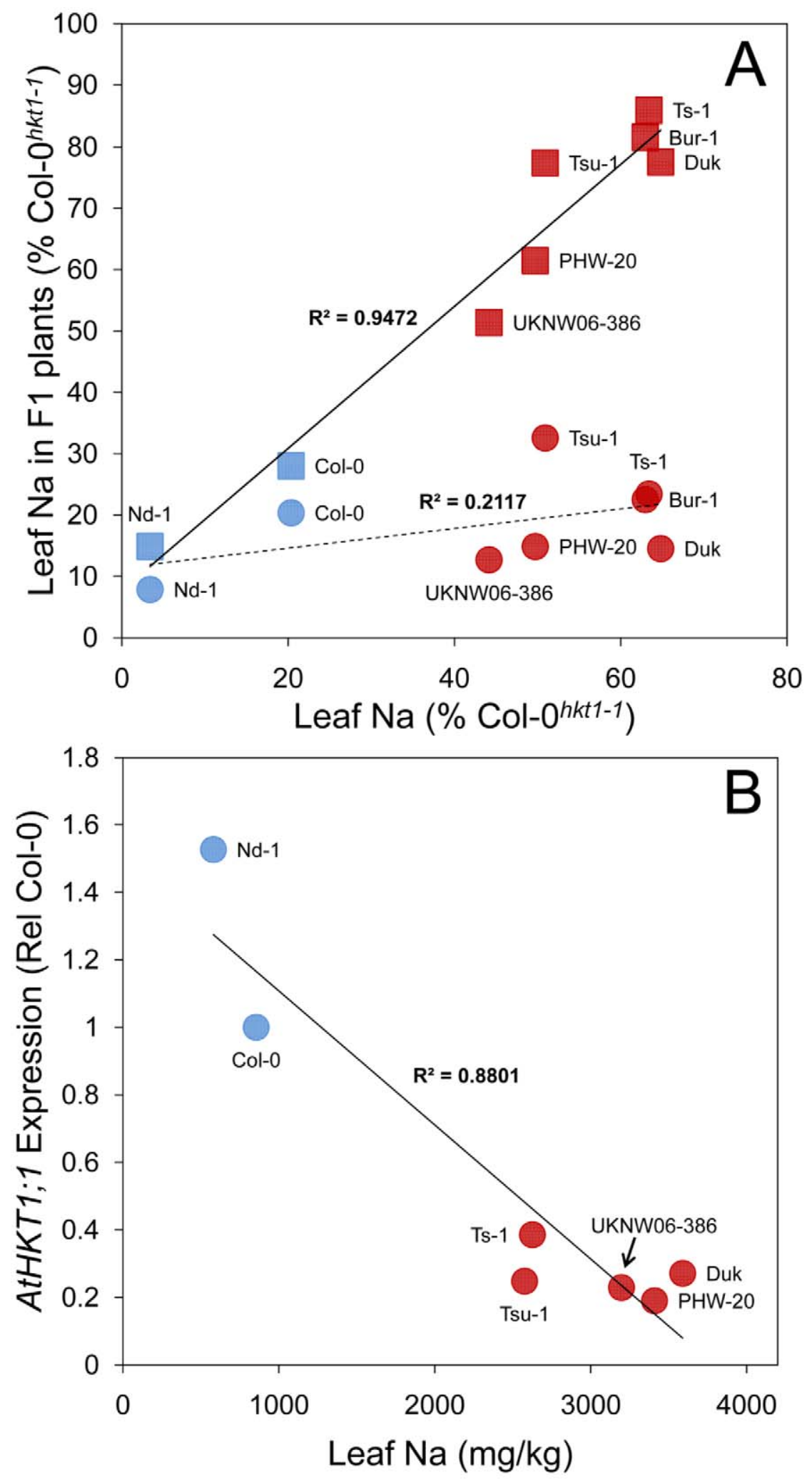

Figure 3. AtHKT1;1 allele strength in various $A$. thaliana accessions assessed by genetic complementation and expression level. A. Leaf $\mathrm{Na}^{+}$accumulation of F1 plants generated from crosses of various accessions to either Col- $0^{\text {hkt } 1-1}$ (squares) or Col- $0^{\mathrm{HKT} 1}$ (circles) compared to leaf $\mathrm{Na}^{+}$accumulation of the non-Col parent of the cross, and represented as a percentage of leaf $\mathrm{Na}^{+}$accumulation of Col- $0^{\text {hkt } 1-1}$. Solid line represents linear regression of data represented by square symbols, dashed line represents linear regression of data represented by circular symbols. B. Root expression level of AtHKT1;1 in various accessions as determined by qRT-PCR, represented as relative to AtHKT1;1 expression in Col-0, and compared to leaf $\mathrm{Na}^{+}$accumulation. Red symbols genotype at Chr4:6392276=T, blue symbols genotype at Chr4:6392276=C. doi:10.1371/journal.pgen.1001193.g003 
the AtHKT1;1 allele frequency in A. thaliana populations and their growth on potentially saline impacted soils (Figure 1A and 1B). Further, we have confirmed by GWA mapping, experimental complementation crosses, and gene expression studies, that this allelic variation directly causes changes in the clinally varying leaf $\mathrm{Na}^{+}$accumulation phenotype via cis-regulatory polymorphisms (Figure 2 and Figure 3). And, finally, we have previously established that the weak AtHKT1;1 alleles we show to be associated with potentially saline soils, are also linked to elevated salinity tolerance [27], providing a plausible mechanistic link between selection for growth on saline soils and variation in AtHKT 1; 1 allele frequency. Such discoveries provide tantalizing evidence that points to selection acting at AtHKT1;1 in natural populations of $A$. thaliana in adaptation to growth in saline environments.

\section{Materials and Methods}

\section{Plant Material and Plant Growth Conditions}

Plants were grown in a controlled environment with $10 \mathrm{~h}$ light/ $14 \mathrm{~h}$ dark $\left(90 \mu \mathrm{mol} \mathrm{m} \mathrm{s}^{-2}\right.$ photosynthetically active light) and 19 to $22^{\circ} \mathrm{C}$, as previously described [31]. Briefly, seeds were sown onto moist soil (Promix; Premier Horticulture) in $10.5^{\prime \prime} \times 21^{\prime \prime} 20$ row trays with various elements added to the soil at subtoxic concentrations (As, Cd, Co, Li, Ni, Rb, and Se [31]) and the tray placed at $4^{\circ} \mathrm{C}$ for 3 days to stratify the seeds and help synchronize germination. Each tray contained 108 plants, six plants each from 18 accessions, with three plants of each accession planted in two different parts of the tray. Each tray contained four common accessions (Col-0, Cvi-0, Fab-2 and Ts-1) used as controls, and 14 test accessions. Trays were bottom-watered twice per week with 0.25 -strength Hoagland solution in which Fe was replaced with $10 \mu \mathrm{M}$ Fe-HBED[N,N'-di(2-hydroxybenzyl)ethylenediamine-N, $\mathrm{N}^{\prime}$-diacetic acid monohydrochloride hydrate; Strem Chemicals, Inc.). After 5 weeks plants were non-destructively sampled by removing one or two leaves and the elemental composition of the tissue analyzed by Inductively Couple Plasma Mass Spectroscopy (ICP-MS). The plant material was rinsed with $18 \mathrm{M} \Omega$ water and placed into Pyrex digestion tubes. For complementation experiments plants were crossed to Col-0 or Col- $0^{h k t 1-1}$ and approximately $12 \mathrm{~F} 1$ plants were grown in the conditions described above.

\section{Mapping Population}

A set of $360 \mathrm{~A}$. thaliana accessions were selected from 5,810 worldwide accessions to minimizing redundancy and close family relatedness, based on the genotypes at 149 SNPs developed in a previous study [32]. Figure S1 and Table S1 show the genetic variation in the core set of 360 accessions vs. a random set of 360 accessions chosen from the genotyped 5,810 accessions. From the selected core set of 360 accessions a subset of 349 were phenotyped using ICP-MS, and of these 337 were genotyped using the Affymetrix SNP-tilling array Atsnptilel which contains probe sets for 248,584 SNPs. Details of the SNP-tilling array and methods for array hybridization and SNP-calling are the same as previously described [24]. In brief, approximately $250 \mathrm{ng}$ of genomic DNA was labeled using the BioPrime DNA labeling system (Invitrogen) and $16 \mu \mathrm{g}$ of the labelled product hybridized to each array. SNPs were called using the Oligo package after slight modifications. Quality control (QG) of the genotypes, and imputation of the missing SNPs were performed following the procedure previously described [24], except that a 15\% mismatch rate was used to filter out low quality arrays. After QC and imputation, the 337 accessions had genotypes for at least 213,497 SNPs. The core set of 360 accessions selected are all available from the Arabidopsis Biological Resource Center (http://abrc.osu.edu/), and the SNP genotypes for the 337 accessions used for the GWA study are available from http://borevitzlab.uchicago.edu/resources/genetic/ hapmap/BaxterCore/.

\section{Tissue Elemental Analysis}

Samples were analyzed as described by Lahner et al. [31]. Tissue samples were dried at $92^{\circ} \mathrm{C}$ for $20 \mathrm{~h}$ in Pyrex tubes $(16 \times 100 \mathrm{~mm})$ to yield approximately $2-4 \mathrm{mg}$ of tissue for elemental analysis. After cooling, seven of the 108 samples from each sample set were weighed. All samples were digested with $0.7 \mathrm{ml}$ of concentrated nitric acid (OmniTrace; VWR Scientific Products), and diluted to $6.0 \mathrm{ml}$ with $18 \mathrm{M} \Omega$ water. Elemental analysis was performed with an ICP-MS (Elan DRCe; PerkinElmer) for Li, B, Na, Mg, P, S, K, $\mathrm{Ca}, \mathrm{Mn}, \mathrm{Fe}, \mathrm{Co}, \mathrm{Ni}, \mathrm{Cu}, \mathrm{Zn}, \mathrm{As}$, Se, Rb, Mo, and Cd. A liquid reference material composed of pooled samples of $A$. thaliana leaves was run every $9^{\text {th }}$ sample to correct for ICP-MS run to run variation and within-run drift. All samples were normalized to the calculated weights, as determined with an iterative algorithm using the best-measured elements, the weights of the seven weighed samples, and the solution concentrations, implemented in the Purdue Ionomics Information Management System (PiiMS) [33] (for a full description see www.ionomicshub.org). Data for all elements is available for viewing and download at www. ionomicshub.org in trays 1478-1504.

\section{Quantification of AtHKT1;1 mRNA}

To quantify the levels of AtHKT1;1 mRNA in roots of the various accessions studied, we used a protocol similar to that of Rus et al. [27]. Roots from plants grown under identical conditions to those used for ICP-MS analysis were separated from the shoots and rinsed thoroughly with deionized water to remove any soil contamination. The samples were frozen in liquid nitrogen and stored at $-80^{\circ} \mathrm{C}$ until extraction. Total RNA was extracted, and DNase digestion was performed during the extraction, using the Invitrogen PureLink RNA Mini Kit. Two micrograms of total RNA were used as a template to synthesize first-strand cDNA with random hexamers, using SuperScript II Reverse Transcriptase (Invitrogen Life Technologies). Quantitative real-time PCR (qRTPCR) was performed with first strand cDNA as a template on four technical replicates from three independent biological samples for each accession, using a sequence detector system (StepOne Plus, Applied Biosystems). For normalization across samples within a qRT-PCR run the expression of the Actin 1 gene (At2g37620) was used with the following primers: CPRD66, 5'-TGG AAC TGG AAT GGT TAA GGC TG-3' and CPRD67, 5' -TCT CGA GAG TCG AGC ACA ATA C-3'. For quantification of AtHKT1;1 the following primers were used: HKT-RTF, 5'-TGG GAT CTT ATA ATT CGG ACA GTT C-3' and HKT-RTR, 5'-GAT AAG ACG CTC GCG ATA ATC AGT-3'. The fold induction relative to AtHKT1; 1 expression in Col-0 roots was calculated following the method of Livak and Schmittgen [34]. CT values were determined based on efficiency of amplification. The mean CT values were normalized against the corresponding Actin 1 gene and $\triangle \mathrm{CT}$ values calculated as $\mathrm{CT}_{\text {AtHKT 1;1 }}-\mathrm{CT}_{\text {Actin }}$ 1. The expression of AtHKT 1; 1 was calculated using the $2^{(\Delta \mathrm{CT})}$ method [34]. To normalize between samples analyzed in separate qRT-PCR runs, we divided the $\Delta \mathrm{CT}$ for each line by the $\Delta \mathrm{CT}$ of Col-0 roots in that run.

\section{Data Analysis}

ICP-MS measurements below zero and extreme outliers (those values that were greater than the $90^{\text {th }}$ percentile $+2 \times\left(90^{\text {th }}-10^{\text {th }}\right.$ 
percentile) within each tray were removed. To account for variation in the growth environment, the four control accessions included in each tray were used to create a tray specific normalization factor. Briefly, for each element, each control accession in a given tray was compared to the overall average for that accession across all trays to obtain an element $\times$ line $\times$ tray specific normalization factor. The four element $\times$ line $\times$ tray factors in a give tray were then averaged to create a tray $\times$ element normalization factor for the tray. Every value for the element in the tray was then multiplied by the normalization factor. See Figure S2 for data of control accessions before and after the normalization. The mean of each accession was then used for all subsequent analysis. Normalized $\mathrm{Na}^{+}$values and their frequency distribution can be found in Dataset S1 and Figure S3.

Genotype calls for all 349 accessions were obtained using the methods previously described [24]. GWA analysis was done with correction for confounding using a mixed-model that uses a genetic random effect with a fixed covariance structure to account for population structure [25] implemented in the program EMMA [24]. The contribution of the best performing SNP $(\mathrm{C}$ or $\mathrm{T}$ at Chr4:6392276 =is T) was checked using un-normalized $\mathrm{Na}^{+}$data and the linear model:

$$
N a=\mu+i s T+\varepsilon, \varepsilon \stackrel{i n d}{\sim} N\left(0, \sigma^{2}\right)
$$

using the $l m$ and anova functions from $\mathrm{R} v 2.9 .1$. The control accessions were excluded from this analysis. The output of the statistical model can be found in Text S1. Although the samples were nested in trays, Figure S4 indicates that the best performing SNP is essentially evenly distributed across all trays.

The geographical location of each accession was obtained from TAIR (www.arabidopsis.org). When processing the original data, we found an inconsistency for one of the high-Na accessions, CS28373 (also known as Jm-1). The listed latitude and longitude $(49,15)$ of the accession do not match the location name "Jamolice" from where this accession was collected. The town Jamolice is located at 49.0721283 latitude and 16.2532139 longitude (http://www.gpsvisualizer.com/geocode). In the interests of consistency, we used the original coordinates, although altering the location did not materially change the analysis. The distance to the coast or saline/sodic areas was calculated by obtaining the longitudes and latitudes of the shoreline/coast from the National Oceanic and Atmospheric Administration's National Geophysical Data Center (NOAAs NGDG http://www.ngdc. noaa.gov/ngdc.html) and the saline and sodic soils data from the European Soils database [23]. The pointDistance function in $\mathrm{R}$ 2.10.0 and the package raster were used to calculate the Greatcircle distance to the shoreline or saline/sodic areas. We created a variable (toSeaSal) representing the shortest distance from the target accession to the shoreline/coast or saline/sodic area. The accession coordinates, distance to sea, distance to saline environment and SNP genotype at Chr4:6392276 can all be found in Dataset S1.

The method used to collect accessions and assemble the population might introduce unintended confounding effects that violate the assumption of independent locations used by our models. To determine whether the locations of the accessions were spatially dependent we performed a Mantel test [35] on the distances from the 300 accessions to the coast or known saline/ sodic areas. The simulated p-values of 50 permutations tests with 999 repeatedly simulated samples are 0.996 , indicating that an assumption of independency for the response variable toSeaSal is acceptable.
To test for associations between leaf $\mathrm{Na}^{+}(\mathrm{Na})$, genotype at the highest scoring SNP ( $\mathrm{C}$ or $\mathrm{T}$ at Chr4:6392276=isT), and the distance to the nearest coast or saline/sodic area (toSeaSal), we used the package $l m$ in $\mathrm{R} 2.10 .0$ to fit linear models, with the weights determined by the following approach. First, to quantify the strength of the relationship between toSeaSal and the leaf sodium $\mathrm{Na}$, we fit the data into a linear model and regressed toSeaSal on $\mathrm{Na}$.

$$
\text { toSeaSal }=\beta_{0}+\beta_{1}(\mathrm{Na})+\varepsilon, \text { where } \varepsilon \stackrel{\text { ind }}{\sim} \mathrm{N}\left(0, \sigma_{(\mathrm{Na})}^{2}\right)
$$

Second, we applied a regression approach to single-factor analysis [36] between toSeaSal and is T and tested if the average distance to coast or saline/sodic areas of samples having the high $\mathrm{Na} \mathrm{T}$ allele is significantly different from the average of samples having the $\mathrm{C}$ allele.

$$
\text { toSeaSal }=\mu+\tau_{1}(\text { isT })+\varepsilon, \text { where } \varepsilon \stackrel{\text { ind }}{\sim} \mathrm{N}\left(0, \sigma_{(\text {isT })}^{2}\right)
$$

Finally, we regressed toSeaSal on the interaction between $\mathcal{N a}$ and is $T$ to inspect how the two predictors jointly affect the distance to sea or saline/sodic.

$$
\begin{aligned}
\text { toSeaSal }= & \mu+\tau_{1}(\text { is } \mathrm{T})+\beta_{1}(\mathrm{Na})+\beta_{2}(\text { is } \times \mathrm{Na}) \\
& +\varepsilon, \text { where } \varepsilon \stackrel{\text { ind }}{\sim} \mathrm{N}\left(0, \sigma_{(\mathrm{isT} \times \mathrm{Na})}^{2}\right)
\end{aligned}
$$

To perform the significance tests on the linear coefficients, $\mathrm{Na}$ should be centered at the mean [36]. The extent of variation of distances to saline environments changes with both leaf $\mathrm{Na}^{+}$ concentrations and genotypes (Figure S5). Therefore, all three models account for this heterogeneity of variation, and parameters of the models are fitted using weighted least squares. The variances of the error terms in equation 2, 3, and 4 are not constant, and are related to the predictors according to the diagnosis on the model residuals. The models were fit using iterative weighted least squares [36]. In addition to the parametric test (model 3), we performed a non-parametric test (Wilcoxon rank-sum test or Wilcoxon-MannWhitney test [37]) using the wilcox.test function in R package stats, to assess whether toSeaSal is higher in the lines with the $\mathrm{T}$ allele than those with the $\mathrm{C}$ allele at Chr4:6392276. The p-value of the Wilcoxon rank-sum test is 0.006224 indicating that both the parametric and non-parametric approaches reach the same conclusion. The statistical output of all models can be found in Text S1.

\section{Supporting Information}

Dataset S1 Data set containing accession name, ID, country and origin, lat and long of collection site, leaf $\mathrm{Na}, \mathrm{SNP}$ at C4_6392276, toSeaSal, toSea, and toSal.

Found at: doi:10.1371/journal.pgen.1001193.s001 (0.09 MB XLS)

Figure S1 Pairwise genetic distance for the core 360.

Found at: doi:10.1371/journal.pgen.1001193.s002 (0.85 MB PDF)

Figure S2 Variation in control lines across trays. The average $\mathrm{Na}^{+}$levels of the four control lines (Col-0, Cvi-0, Fab-2 and Ts-1) as well as the average of all other lines in each tray is plotted before (A) and after (B) normalization.

Found at: doi:10.1371/journal.pgen.1001193.s003 (0.25 MB PPTX)

Figure S3 The distribution of average leaf $\mathrm{Na}^{+}$values across all accessions used for association mapping. The $\mathrm{x}$-axis displays the 
parts per million (ppm) $\mathrm{Na}^{+}$values before normalization. The barbell symbols indicate the range of the $\mathrm{Na}$ values for the four control accessions across the 26 trays of the experiment.

Found at: doi:10.1371/journal.pgen.1001193.s004 (0.10 MB PPTX

Figure S4 Distribution of Chr4:6392276 across experiments. The lines containing the high $\mathrm{Na}^{+}$allele at the SNP most significantly correlated with $\mathrm{Na}$ (Chr4:6392276) are approximately evenly distributed among the 26 trays of the experiment. Bars indicate the number of accessions having either the $\mathrm{T}$ allele (blue) or the $\mathrm{C}$ allele (red) at the SNP most significantly correlated with $\mathrm{Na}^{+}, \mathrm{Chr} 4: 6392276$.

Found at: doi:10.1371/journal.pgen.1001193.s005 (0.16 MB PPTX)

Figure S5 Scatter plot of distance to sea or saline/sodic areas (toSeaSal) versus leaf $\mathrm{Na}$. The red colored $\mathrm{T}$ corresponds to the samples having a $\mathrm{T}$ at Chr4:6392276. The blue colored $\mathrm{C}$ represents samples having a $\mathrm{C}$ at Chr4:6392276. The black line is a lowess curve fitting all the 300 samples. The red line is a lowess

\section{References}

1. Wilczek AM, Roe JL, Knapp MC, Lopez-Gallego C, Martin LJ, et al. (2009) Effects of genetic perturbation on seasonal life history plasticity. Science 323: 930-934.

2. Paaby AB, Blacket MJ, Hoffmann AA, Schmidt PS (2010) Identification of a candidate adaptive polymorphism for Drosophila life history by parallel independent clines on two continents. Mol Ecol 19: 760-774.

3. McKechnie SW, Blacket MJ, Song SV, Rako L, Carroll X, et al. (2010) A clinally varying promoter polymorphism associated with adaptive variation in wing size in Drosophila. Mol Ecol 19: 775-784.

4. Storz JF, Runck AM, Sabatino SJ, Kelly JK, Ferrand N, et al. (2009) Evolutionary and functional insights into the mechanism underlying highaltitude adaptation of deer mouse hemoglobin. Proc Natl Acad Sci U S A 106: $14450-14455$

5. Brady K, Kruckeberg A, Bradshaw HJ (2005) Evolutionary ecology of plant adaptation to serpentine soils. Annual Review of Ecology, Evolution, and Systematics 36: 243-266.

6. Lowry DB, Rockwood RC, Willis JH (2008) Ecological reproductive isolation of coast and inland races of Mimulus guttatus. Evolution 62: 2196-2214.

7. Welch ME, Rieseberg L (2002) Habitat divergence between a homoploid hybrid sunflower species, Helianthus paradoxus (Asteraceae), and its progenitors. Am J Bot 89: 472.

8. Macnair MR (1993) The genetics of metal tolerance in vascular plants. New Phytol 124: 541-559.

9. Lexer G, Welch ME, Durphy JL, Rieseberg LH (2003) Natural selection for salt tolerance quantitative trait loci (QTLs) in wild sunflower hybrids: implications for the origin of Helianthus paradoxus, a diploid hybrid species. Mol Ecol 12: 1225-1235.

10. Lowry DB, Hall MC, Salt DE, Willis JH (2009) Genetic and physiological basis of adaptive salt tolerance divergence between coastal and inland Mimulus guttatus. New Phytol 183: 776-788.

11. Hoffmann MH (2002) Biogeography of Arabidopsis thaliana (L.) Heynh. (Brassicaceae). J Biogrogr 29: 125-134

12. Alonso-Blanco C, Aarts MG, Bentsink L, Keurentjes JJ, Reymond M, et al. (2009) What has natural variation taught us about plant development, physiology, and adaptation? Plant Cell 21: 1877-1896.

13. Mitchell-Olds T, Schmitt J (2006) Genetic mechanisms and evolutionary significance of natural variation in Arabidopsis. Nature 441: 947-952.

14. Munns R, Tester M (2008) Mechanisms of salinity tolerance. Annu Rev Plant Biol 59: 651-681.

15. Edelist C, Lexer C, Dillmann C, Sicard D, Rieseberg LH (2006) Microsatellite signature of ecological selection for salt tolerance in a wild sunflower hybrid species, Helianthus paradoxus. Mol Ecol 15: 4623-4634.

16. Karrenberg S, Edelist C, Lexer C, Rieseberg L (2006) Response to salinity in the homoploid hybrid species Helianthus paradoxus and its progenitors $H$. annuus and H. petiolaris. New Phytol 170: 615-629.

17. Lexer C, Lai Z, Rieseberg LH (2004) Candidate gene polymorphisms associated with salt tolerance in wild sunflower hybrids: implications for the origin of Helianthus paradoxus, a diploid hybrid species. New Phytol 161: 225-233.

18. Lexer G, Welch ME, Raymond O, Rieseberg LH (2003) The origin of ecological divergence in Helianthus paradoxus (Asteraceae): selection on transgressive characters in a novel hybrid habitat. Evolution 57: 1989-2000.

19. Franzén LG (1990) Transport, deposition and distribution of marine aerosols over southern Sweden during dry westerly storms. Ambio 19: 180-188. curve fitting the 21 samples with the $\mathrm{T}$ allele. The blue line is a lowess curve fitting the 279 samples with the $\mathrm{C}$ allele.

Found at: doi:10.1371/journal.pgen.1001193.s006 (0.34 MB PPTX)

Table S1 Comparing the genetic variation between Core360 and Random 360 accessions.

Found at: doi:10.1371/journal.pgen.1001193.s007 (0.04 MB DOC)

Text S1 Output of statistical models.

Found at: doi:10.1371/journal.pgen.1001193.s008 (0.01 MB DOCX)

\section{Author Contributions}

Wrote the paper: DES IB JNB DY OV. Designed and supervised the project: DES. Phenotying the set of 360 accessions and data normalization: IB. Genetic complementation and gene expression studies: JNB. ICP-MS analysis: BL. Plant growth, harvesting, and sample preparation: EY. Performed selection and genotyping of the 360 accessions used for the genome-wide association mapping population: YL JOB JB. GWA analysis: MN YSH. Statistical analysis: OV DY.

20. Goulding KWT, Poulton PR, Thomas VH, Williams RJB (1986) Atmospheric deposition at Rothamsted, Saxmundham, and Woburn experimental stations, England, 1969-1984. Water, Air, \& Soil Pollution 29: 1573-2932.

21. Gustafsson MER, Franzén LG (2000) Inland transport of marine aerosols in Southern Sweden. Atmospheric Environment 34: 313-325.

22. Suzuki T, Iizuka Y, Matsuoka K, Furukawa T, Kamiyama K, et al. (2002) Distribution of sea salt components in snow cover along the traverse route from the coast to Dome Fuji station $1000 \mathrm{~km}$ inland at east Dronning Maud Land, Antarctica. Tellus 54B: 407-411.

23. Tóth G, Montanarella L, Rusco E (2008) Threats to soil quality in Europe EUR 23438- Scientific and Technical Research series Luxembourg: Office for Official Publications of the European Communities: 61-74.

24. Atwell S, Huang YS, Vilhjalmsson BJ, Willems G, Horton M, et al. (2010) Genome-wide association study of 107 phenotypes in Arabidopsis thaliana inbred lines. Nature 465: 627-631.

25. Yu J, Pressoir G, Briggs WH, Vroh BL, Yamasaki M, et al. (2006) A unified mixed-model method for association mapping that accounts for multiple levels of relatedness. Nat Genet 38: 203-208.

26. Horie T, Hauser F, Schroeder JI (2009) HKT transporter-mediated salinity resistance mechanisms in Arabidopsis and monocot crop plants. Trends Plant Sci 14: 660-668.

27. Rus A, Baxter I, Muthukumar B, Gustin J, Lahner B, et al. (2006) Natural variants of AtHKT1 enhance Na+ accumulation in two wild populations of Arabidopsis. PLoS Genet 2: e210. doi:10.1371/journal.pgen.0020210.

28. Moller IS, Gilliham M, Jha D, Mayo GM, Roy SJ, et al. (2009) Shoot Na+ exclusion and increased salinity tolerance engineered by cell type-specific alteration of $\mathrm{Na}+$ transport in Arabidopsis. Plant Cell 21: 2163-2178.

29. Berthomieu P, Conejero G, Nublat A, Brackenbury WJ, Lambert C, et al. (2003) Functional analysis of AtHKT1 in Arabidopsis shows that $\mathrm{Na}(+)$ recirculation by the phloem is crucial for salt tolerance. EMBO J 22: 2004-2014.

30. Maser P, Eckelman B, Vaidyanathan R, Horie T, Fairbairn DJ, et al. (2002) Altered shoot/root $\mathrm{Na}+$ distribution and bifurcating salt sensitivity in Arabidopsis by genetic disruption of the Na+ transporter AtHKT1. FEBS Lett 531: 157-161

31. Lahner B, Gong J, Mahmoudian M, Smith EL, Abid KB, et al. (2003) Genomic scale profiling of nutrient and trace elements in Arabidopsis thatiana. Nat Biotechnol 21: 1215-1221.

32. Platt A, Horton M, Huang YS, Li Y, Anastasio AE, et al. (2010) The scale of population structure in Arabidopsis thaliana. PLoS Genet 6: e1000843. doi:10.1371/journal.pgen.1000843.

33. Baxter I, Ouzzani M, Orcun S, Kennedy B, Jandhyala SS, et al. (2007) Purdue ionomics information management system. An integrated functional genomics platform. Plant Physiol 143: 600-611.

34. Livak KJ, Schmittgen TD (2001) Analysis of relative gene expression data using real-time quantitative PCR and the $2-\Delta \Delta \mathrm{Ct}$ method. Methods 25: 402-408.

35. Mantel N (1967) The detection of disease clustering and a generalized regression approach. Cancer Research 27: 209.

36. Kutner MH, Nachtsheim CJ, Li W, Neter J (2005) in Building the Regression Model III: Remedial Measures Ch. 11, 16, 22, (McGraw Hill).

37. Mann HB, Whitney DR (2947) On a test of whether one of two random variables is stochastically larger than the other. The Annals of Mathematical Statistics 18: 50-60. 